Pacific Journal of Mathematics

A KRASNOSEL'SKIII-TYPE THEOREM FOR UNIONS OF TWO
STARSHAPED SETS IN THE PLANE

MARILYN BREE N 


\title{
A KRASNOSEL'SKII-TYPE THEOREM FOR UNIONS OF TWO STARSHAPED SETS IN THE PLANE
}

\author{
MARILYN BREEN
}

Let $S$ be a simply connected polygonal region in the plane, symmetric with respect to the $x$ and $y$ axes, such that each edge of $S$ is parallel to one of these axes. Assume that for every set $E$ consisting of 6 or fewer edges of $S$ there exist points $t_{1}$ and $t_{2}$ collinear with the origin (and depending on $E$ ) such that every point in $\bigcup\{e: e$ in $E\}$ is visible via $S$ from $t_{1}$ or $t_{2}$ (or both). Then $S$ is a union of two starshaped sets. The number 6 is best possible.

Furthermore, an example reveals that there is no finite Krasnosel'skii number which characterizes arbitrary unions of two or more starshaped sets in the plane.

1. Introduction. We begin with some preliminary definitions. Let $S$ be a set in $R^{d}$. For points $x$ and $y$ in $S$, we say $x$ sees $y$ via $S$ ( $x$ is visible from $y$ via $S$ ) if and only if the corresponding segment $[x, y]$ lies in $S$. Point $x$ is clearly visible from $y$ via $S$ if and only if there is some neighborhood $N$ of $x$ such that $y$ sees each point of $N \cap S$ via $S$. Set $S$ is starshaped if and only if there is some point $p$ in $S$ such that $p$ sees each point of $S$ via $S$, and the set of all such points $p$ is called the (convex) kernel of $S$.

A well-known theorem of Krasnosel'skii [5] states that if $S$ is a nonempty compact set in $R^{d}$, then $S$ is starshaped if and only if every $d+1$ points of $S$ are visible via $S$ from a common point. Further, points of $S$ may be replaced by boundary points of $S$ to produce a stronger result. An interesting problem related to this concerns obtaining a Krasnosel'skii-type theorem for unions of starshaped sets in the plane $R^{2}$. This kind of problem is mentioned in [10, Prob. 6.6, p. 178] and in [1]. Moreover, using work by Lawrence, Hare, and Kenelly [7] concerning unions of convex sets, the following Krasnosel'skii-type results for unions of starshaped sets are obtained in [2]: (1) For $S$ compact in $R^{2}, S$ is a union of two starshaped sets if for every finite set $F$ in the boundary of $S$ there exist points $s$ and $t$ (depending on $F$ ) such that each point of $F$ is clearly visible via $S$ from at least one of $s$ or $t$. If in addition set $S$ is simply connected, then 'clearly visible' may be replaced by the weaker 
term 'visible'. (2) In general, for $S$ a compact set in some linear topological space, $S$ is a union of $k$ starshaped sets if and only if for every finite set $F$ in $S$ there exist points $s_{1}, \ldots, s_{k}$ (depending on $F$ ) such that each point of $F$ sees via $S$ at least one of the $s_{i}$ points. Unfortunately, the finiteness condition in (2) above cannot be improved, and no finite Krasnosel'skii number exists to characterize arbitrary unions of two or more compact starshaped sets, even in the plane. (See Example 4.)

Still, the problem of obtaining a finite Krasnosel'skii number for certain families of sets remains open. Since the Helly number $d+1$ plays a fundamental role in the classical Krasnosel'skii theorem, we would expect the piercing number in [4] to be important in any kind of generalization. Although an example from [5, pp. 11-12] reveals that no finite $n$-piercing number exists for arbitrary families of compact sets when $n \geq 2$, recent work by Danzer and Grünbaum [3] reveals that such a piercing number does exist for families of boxes. In a similar spirit, Toussaint and El-Gindy [9] have shown that for polygonal regions whose edges are parallel to the coordinate axes, the classical Krasnosel'skii number 3 may be reduced to 2 . Therefore, it seems reasonable to attempt to establish a generalized Krasnosel'skii theorem for such sets. This is the problem considered here.

The following terminology will be used throughout the paper: Conv $S$, int $S$, rel int $S$, bdry $S$, and ker $S$ will denote the convex hull, interior, relative interior, boundary, and kernel, respectively, for set $S$. For distinct points $x$ and $y, L(x, y)$ will represent the line through $x$ and $y$ and $\frac{1}{2}(x+y)$ will denote the midpoint of segment $[x, y]$. The reader is referred to Valentine [10] and to Lay [8] for a discussion of these concepts.

2. Preliminary results. We start with an easy lemma.

LEMMA 1. Let $S$ be a compact, simply connected set in $R^{2}$, with $a, b \in S$. If each boundary point of $S$ sees via $S$ either $a$ or $b$, then each point of $S$ sees via $S$ either $a$ or $b$.

Proof. Let $x \in S$ to show $x$ sees via $S$ either $a$ or $b$. If $x \in$ bdry $S$, the result is immediate, so assume that $x \in$ int $S$. Suppose $[a, x] \nsubseteq S$, and let $\left[a_{1}, a_{2}\right]$ be the component of $S \cap L(a, x)$ which contains $x$. Clearly $a_{1}<x<a_{2}, a_{1}$ and $a_{2}$ are in bdry $S$, and $a$ sees via $S$ neither $a_{1}$ nor $a_{2}$. Hence $b$ sees via $S$ both $a_{1}$ and $a_{2}$. Since $S$ is simply connected, $\operatorname{conv}\left\{b, a_{1}, a_{2}\right\} \subseteq S,[b, x] \subseteq S$, and the lemma is established.

We make several observations concerning Lemma 1. 
First, if $S$ is not required to be simply connected, then the result in Lemma 1 fails and in fact $S$ is not necessarily a union of two starshaped sets. This is illustrated in [2, Example 1].

Next, it is interesting to notice that even when $S$ is a polygonal region satisfying additional hypotheses, bdry $S$ cannot be replaced by the vertex set of $S$ in Lemma 1. This is demonstrated in Example 1 below.

EXAMPLE 1. Let $S$ be the polygonal region in Figure 1, with each edge of $S$ parallel to one of the coordinate axes. Every vertex of $S$ is clearly visible via $S$ from either point $a$ or point $b$, yet $S$ is not a union of two starshaped sets. In fact, no point of $S$ sees via $S$ two members of $\{p, q, r\}$.

Finally, while the result in Lemma 1 holds for two points, it fails for three, as Example 2 illustrates.

EXAMPLE 2. Let $S$ be the simply connected set in Figure 2. Every boundary point of $S$ is visible via $S$ from one of the points $a, b, c$. However, interior point $x$ sees none of these points via $S$, and $S$ is not a union of three starshaped sets.

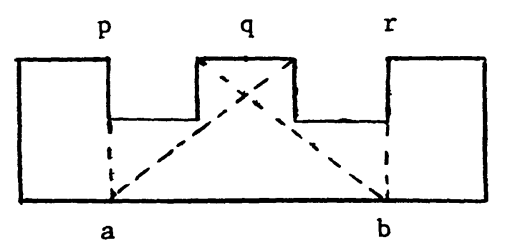

Figure 1

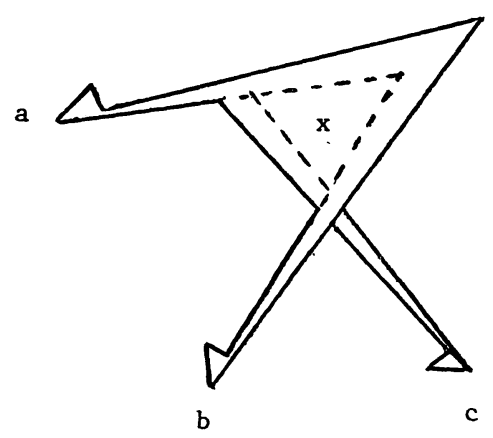

FIGURE 2 
3. The main theorem. We will be concerned with the proof of the following theorem.

THEOREM 1. Let $S$ be a simply connected polygonal region in the plane, symmetric with respect to the $x$ and $y$ axes, such that each edge of $S$ is parallel to one of these axes. Assume that for every set E consisting of 6 or fewer edges of $S$ there exist points $t_{1}$ and $t_{2}$ collinear with the origin $\theta$ (and depending on $E$ ) such that every point in $\bigcup\{e$ : $e$ in $E\}$ is visible via $S$ from $t_{1}$ or $t_{2}$ (or both). Then $S$ is a union of two starshaped sets. The number 6 is best possible.

Proof. By Lemma 1, it suffices to show that there exist points $t, t^{\prime}$ in $S$ such that every boundary point of $S$ sees via $S$ either $t$ or $t^{\prime}$. As in [9], it is convenient to order bdry $S$ in a clockwise direction. It is clear that this order, in turn, induces a natural order on each edge of $S$. Since the edges of $S$ are parallel to the coordinate axes, we may use the terminology employed in [9] to classify each horizontal edge of $S$ as a 'right' or a 'left' edge and to classify each vertical edge of $S$ as an 'up' or a 'down' edge, according to the order it inherits from bdry $S$.

Observe that relative interior points of 'right' edges see via $S$ no points above their corresponding lines. Similar statements can be made for 'left' edges and points below, 'up' edges and points to their left, 'down' edges and points to their right.

Select a 'right' edge $e_{R}$ whose $y$ coordinate is as small as possible and a 'left' edge $e_{L}$ whose $y$ coordinate is as large as possible. Let $R$ and $L$ denote the lines determined by $e_{R}$ and $e_{L}$, respectively. Similarly, select 'up' edge $e_{U}$ whose $x$ coordinate is as large as possible and 'down' edge $e_{D}$ whose $x$ coordinate is as small as possible, and let $U$ and $D$ denote their associated lines. By an observation above, points of rel int $e_{R}$ see via $S$ only points on or below $R$. Of course, parallel statements hold for remaining edges $e_{L}, e_{U}, e_{D}$ and corresponding lines. By symmetry of $S$, clearly $U \neq D$ and $R \neq L$.

In case line $L$ is below line $R$ and line $D$ is to the right of line $U$, then it is easy to show that $S$ is starshaped and $\operatorname{ker} S$ is exactly the rectangular region bounded by these four lines. In case line $L$ is below line $R$ and line $D$ is to the left of line $U$, the argument is equivalent to Case 1 below. Hence throughout the remainder of the proof we will assume that line $L$ is above line $R$. For convenience of notation, let $M$ (middle) denote the closed subset of $S$ bounded by lines $L$ and $R$. Let $T$ (top) be the closed subset of $S$ whose points are on or above $L$, and let $B$ (bottom) be the 
closed subset of $S$ whose points are on or below $R$. For future reference, observe that each edge of $S$ is a subset of at least one of $M, T$, or $B$.

The following notation will be helpful: Let $\xi$ denote symmetry in the $x$ axis and let $\eta$ denote symmetry in the $y$ axis. Let $L+$ and $L-$ represent closed halfplanes bounded by $L$ with int $L$ - containing no 'left' edge of bdry $S$. Similarly, define halfplanes $U+, U-$ (with int $U-$ containing no 'up' edge), $R+, R-, D+, D-$. Note that $U, U+$, $U$ - are the $\eta$-images of $D, D+, D-$, respectively, while $R, R+, R-$ are the $\xi$-images of $L, L+, L-$.

The proof will be accomplished by considering two cases, determined by the positions of $U$ and $D$.

Case 1. For the moment, assume that line $D$ lies to the right of line $U$.

First we make some observations.

(1) In Case 1, $S$ is convex in horizontal direction. That is, for $x, y \in S$ and $[x, y]$ horizontal, it follows that $[x, y] \subseteq S$. This holds because for each horizontal line $A$, each component of $A \cap S$ must have its left endpoint on some 'up' edge (in $U+$ ) and its right endpoint on some 'down' edge (in $D+$ ). But $D+$ and $U+$ are disjoint, so $A \cap S$ has at most one component. Analogous reasoning shows that $S \cap D-\cap U-$ is convex in vertical direction and contains the rectangular region $D-\cap$ $U-\cap L+\cap R+$.

(2) Each region bounded by a simple closed curve in $S$ is contained in $S$ (since $S$ is simply connected). In particular, if $x$ in $S$ sees via $S$ points $a$ and $b$ of edge $e$, then $x$ sees via $S$ the interval $[a, b]$. Thus (3) below holds.

(3) For $x \in S$ and $e$ any edge of bdry $S$, the subset of $e$ seen by $x$ is a compact interval (possibly empty).

Now let lines $L$ and $R$ meet the $y$ axis at points $p$ and $q$, respectively. We will show that for $e$ any edge in bdry $S$, each point of $e$ sees (always via $S$ ) either $p$ or $q$.

(4) The set bdry $S \cap$ int $L-$ consists of 'up' and 'right' edges in $U+$, one 'right' edge crossing $U-\cap D-$, and 'right' and 'down' edges in $D+$. By (1) and (2), it follows that each point of $S \cap L-$ sees $p$. By $\xi$-symmetry, each point of $S \cap R-\operatorname{sees} q$.

(5) For $e$ in the remaining part $S \cap L+\cap R+$ (and not on $L$ or $R$ ), select $t_{1}, t_{2}$ collinear with $\theta$ to satisfy the hypothesis of the theorem for the edge set

$$
\left\{e, \xi(e), \eta(e), \xi \eta(e), e_{R}, e_{L}\right\}
$$


By definition of $e_{L}$ and $e_{R}$, we may assume $t_{1} \in T=S \cap L-$ and $t_{2} \in B=S \cap R-$.

(6) If $e$ is a 'left' edge, $t_{2}$ cannot see the relative interior of $e$ and of $\eta(e)$, so $t_{1}$ sees the whole of $e$ and of $\eta(e)$. By $\eta$-symmetry of $S, \eta\left(t_{1}\right)$ also sees the whole of $e$ and of $\eta(e)$. By (1) and (2), $\operatorname{conv}\left\{t_{1}, \eta\left(t_{1}\right), e, \eta(e)\right\}$ is in $S$. But this set contains $p$, so $p$ sees each point of $e$. Similarly, if $e$ is a 'right' edge, $q$ sees each point of $e$.

(7) If $e=[a, b]$ is vertical and preceded and followed by 'left' ('right') edges, then by (6), point $p$ (point $q$, respectively) sees $a$ and $b$, so it sees the whole of $e$ by (3).

(8) If $e$ is an 'up' ('down') edge preceded by a 'right' ('left') edge and followed by a 'left' ('right') edge, then $p$ sees the upper endpcint of $e$ and $q$ sees the lower endpoint of $e$ by (6). Hence $\operatorname{conv}\{e, p, q\}$ has its boundary in $S$ and lies in $S$ by (2). This means that each point of $e$ sees $p$ as well as $q$.

(9) For the remaining cases we may assume by $\eta$-symmetry of $S$ that $e$ is a 'down' edge in $D+\cap L+\cap R+$ preceded by a 'right' edge and followed by a 'left' edge. Let $a$ denote the upper endpoint of $e$ and $b$ the lower endpoint of $e$. Then $p$ sees $b$ and $q$ sees $a$ by (6). To complete the proof, by (3) it suffices to show that some point $x_{0}$ of $e$ sees $p$ as well as $q$.

Now each point of $e$ and of $\xi \eta(e)$ sees $t_{1}$ or $t_{2}$, and by (3) there are some $x_{1} \in e$ and some $w_{1} \in \xi \eta(e)$ which see $t_{1}$ as well as $t_{2}$. So $Q \equiv$ $\operatorname{conv}\left\{x_{1}, t_{1}, w_{1}, t_{2}\right\}$ has its boundary in $S$ and is contained in $S$ by (2). In particular, if we let $\left\{p_{1}\right\} \equiv L \cap\left[t_{1}, t_{2}\right]$ and $\left\{q_{1}\right\} \equiv R \cap\left[t_{1}, t_{2}\right]=\xi \eta\left(p_{1}\right)$, then $\operatorname{conv}\left\{x_{1}, p_{1}, q_{1}\right\} \subseteq Q \subseteq S$ and $x_{1}$ sees $p_{1}$ as well as $q_{1}$. We assert that some $x_{2} \in e$ sees $\left\{p_{2}\right\} \equiv \eta\left(p_{1}\right)$ as well as $q_{2} \equiv \eta\left(q_{1}\right)$. In fact by (5) and (3) some $\eta\left(x_{2}\right) \in \eta(e)$ and some $w_{2} \in \xi(e)=\xi \eta \eta(e)$ see $t_{1}$ and $t_{2}$, so by

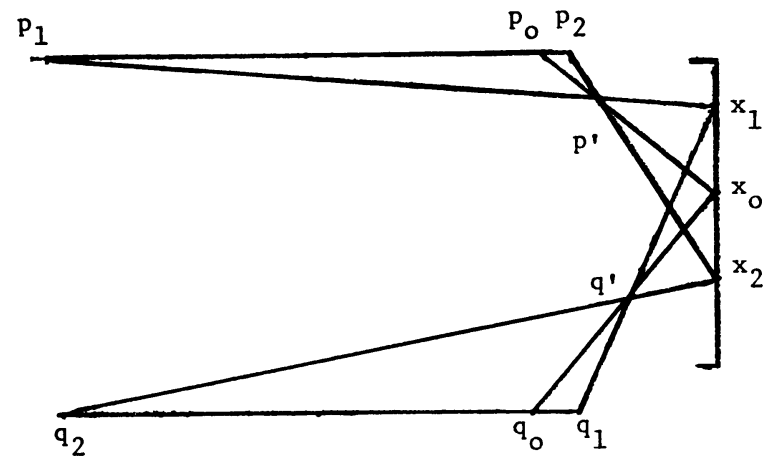

Figure 3

If $x_{0}=\lambda x_{1}+(1-\lambda) x_{2}$ and $\lambda \in[0,1]$, then $\lambda p_{1}+$ $(1-\lambda) p_{2} \in\left[p_{0}, p_{1}\right]$ and $\lambda q_{1}+(1-\lambda) q_{2} \in\left[q_{0}, q_{2}\right]$. 
the previous reasoning $\operatorname{conv}\left\{\eta\left(x_{2}\right), p_{1}, q_{1}\right\}$ is in $S$. It follows by $\eta$-symmetry of $S$ that $\operatorname{conv}\left\{x_{2}, p_{2}, q_{2}\right\} \subseteq S$. In particular, $x_{2} \in e$ sees $p_{2}$ and $q_{2}$.

Note that $p=\frac{1}{2}\left(p_{1}+p_{2}\right)$ and $q=\frac{1}{2}\left(q_{1}+q_{2}\right)$. Now there are two subcases to consider:

In case $\left[x_{1}, p_{1}\right],\left[x_{2}, p_{2}\right]$ intersect and $\left[x_{1}, q_{1}\right],\left[x_{2}, q_{2}\right]$ also intersect, then we have the situation in Figure 3 (up to permutation of indices 1 and 2). There points $p^{\prime}, p_{i}, x_{i}$ are collinear and $q^{\prime}, q_{i}, x_{i}$ are collinear, $i=0,1,2$. The assertion of the caption of Figure 3 is verified by elementary geometry and shows for $\lambda=\frac{1}{2}$ that $p=\frac{1}{2}\left(p_{1}+p_{2}\right) \in\left[p_{0}, p_{1}\right]$ and $q=\frac{1}{2}\left(q_{1}+q_{2}\right) \in\left[q_{0}, q_{2}\right]$. By convexity in horizontal direction, we conclude that $\operatorname{conv}\left\{x_{0}, p_{0}, p, q, q_{0}\right\} \subseteq S$ and in particular that $x_{0} \in e$ sees $p$ and $q$.

In case $\left[x_{1}, p_{1}\right],\left[x_{2}, p_{2}\right]$ do not intersect, neither do $\left[x_{1}, q_{1}\right],\left[x_{2}, q_{2}\right]$. Then conv $\left\{x_{1}, p_{1}, p_{2}, x_{2}\right\}$ and $\operatorname{conv}\left\{x_{1}, q_{1}, q_{2}, x_{2}\right\}$ have their boundaries in $S$. In particular, letting $x_{0} \equiv \frac{1}{2}\left(x_{1}+x_{2}\right) \in e,\left[x_{0}, p\right] \cup\left[x_{0}, q\right] \subseteq S$. Again $x_{0} \in e$ sees $p$ as well as $q$, the desired result.

We conclude that every point of edge $e$ sees via $S$ either $p$ or $q$, and hence every boundary point of $S$ sees via $S$ either $p$ or $q$. Therefore, by Lemma 1, every point of $S$ sees via $S$ either $p$ or $q$, and $S$ is indeed a union of two starshaped sets. The proof for Case 1 is established.

Case 2. Assume that line $D$ lies to the left of line $U$.

The following observations will be helpful.

(1) If $V$ is a vertical line meeting $S$, each component of $V \cap S$ has its top endpoint on a 'right' edge (in $R_{+}$) and its bottom endpoint on a 'left' edge (in $L_{+}$). Thus $L_{-} \cap S$ is convex in vertical direction and contains its orthogonal projection onto $L$. Similarly $U_{-} \cap S$ is convex in horizontal direction and contains its orthogonal projection onto $U$. $\xi$ - and $\eta$-symmetry give corresponding statements on $R_{-} \cap S$ and $D_{-} \cap S$.

For convenience of notation, let $A$ denote the closed rectangular region determined by lines $L, R, D, U$. Let $A_{1}, A_{1}^{\prime}, A_{2}, A_{2}^{\prime}, A_{3}, A_{3}^{\prime}, A_{4}, A_{4}^{\prime}$ denote the eight closed unbounded regions determined by $L, R, D, U$ labeled in a clockwise direction about $A$, with $A_{i}$ meeting $A$ only at the common vertex $a_{i}, 1 \leq i \leq 4$, and with $A_{1}=L_{-} \cap U_{-}$. (See Figure 4.) Then we observe:

(2) $A$ is contained in $S$. In fact, choose points $v_{1}$ and $v_{2}$ which satisfy the hypothesis of Theorem 1 for the collection of edges $\left\{e_{L}, \eta\left(e_{L}\right), e_{R}\right.$, $\left.\eta\left(e_{R}\right), e_{U}, \xi\left(e_{U}\right)\right\}$. From preliminary comments, for $x \in$ (rel int $\left.e_{L}\right) \cup$ (rel int $\left.\eta\left(e_{L}\right)\right), y \in\left(\right.$ rel int $\left.e_{R}\right) \cup\left(\right.$ rel int $\left.\eta\left(e_{R}\right)\right)$, and $z \in\left(\right.$ rel int $\left.e_{U}\right) \cup$ (rel int $\left.\xi\left(e_{U}\right)\right), x$ sees via $S$ at most points of $L_{-}, y$ sees at most points of $R_{-}$, 
$z$ sees at most points of $U_{-}$. Hence for an appropriate labeling, we have $v_{1} \in L_{-}$and each point of $e_{L} \cup \eta\left(e_{L}\right)$ sees $v_{1}, v_{2} \in R_{-}$and each point of $e_{R} \cup \eta\left(e_{R}\right)$ sees $v_{2}$. Furthermore, by the collinearity of $v_{1}, \theta, v_{2}$, one and only one of the points $v_{1}, v_{2}$ sees $e_{U} \cup \xi\left(e_{U}\right)$. If this point is $v_{1}$, then $v_{1} \in A_{1}=L_{-} \cap U_{-}$and $v_{1}$ sees $e_{L}, \eta\left(e_{L}\right), e_{U}, \xi\left(e_{U}\right)$ via $S$. By observation (1) it follows that $S$ contains the subset of bdry $A$ lying in the first quadrant. By the symmetry of $S$, bdry $A \subseteq S$, and since $S$ is simply connected, $A \subseteq S$. Similarly, we can conclude $A \subseteq S$ if $v_{2}$ sees $e_{U} \cup \xi\left(e_{U}\right)$.

From observations (1) and (2) we get:

(3) If $V$ is a vertical line in $D_{+} \cap U_{+}$, then $V \cap S$ is a segment. Hence $D_{+} \cap U_{+} \cap S$ is convex in vertical direction. Similarly, $L_{+} \cap R_{+} \cap S$ is convex in horizontal direction.

For future reference, observe that since $A \subseteq S$, no relative interior point of any edge $e_{L}, e_{R}, e_{U}, e_{D}$ can meet $A$. Therefore, by the symmetry of $S$, we may assume that these edges are labeled so that $e_{L} \subseteq A_{1}$, $e_{U} \subseteq A_{2}, e_{R} \subseteq A_{3}$, and $e_{D} \subseteq A_{4}$. Thus $\eta\left(e_{L}\right) \subseteq A_{4}, \xi\left(e_{U}\right) \subseteq A_{1}, \eta\left(e_{R}\right) \subseteq$ $A_{2}$, and $\xi\left(e_{D}\right) \subseteq A_{3}$.

To complete the proof in Case 2, we will show that each boundary point $d$ of $S$ sees via $S$ two consecutive points from $\left(a_{1}, a_{2}, a_{3}, a_{4}, a_{1}\right)$, the vertex set of $A$. Let $e$ be an edge of $S$ containing point $d$. Clearly $e$ lies in one of the closed halfplanes determined by each line $L, R, D$, and $U$. Since $A \subseteq S$, either $e \subseteq A_{i}$ or $e \subseteq A_{i}^{\prime}$ for some $i, 1 \leq i \leq 4$. First consider the situation in which $e \subseteq A_{i}$ for some $i$, and for convenience of notation, assume $i=1$. Choose points $t_{1}$ and $t_{2}$ satisfying the hypothesis of Theorem 1 for $\left\{e, \eta(e), e_{L}, e_{U}, e_{R}, e_{D}\right\}$.

Using earlier comments, it is clear that for an appropriate labeling of $t_{1}$ and $t_{2}$, one of the following must occur: Either $t_{1} \in A_{4}$ and $t_{2} \in A_{2}$ or $t_{1} \in A_{1}$ and $t_{2} \in A_{3}$. In the first case, using observation (1), each point of $e$ which sees $t_{1}$ must see $a_{4}$ and $a_{1}$ as well. Each point of $e$ which sees $t_{2}$ must see $a_{1}$ and $a_{2}$. Thus point $d$ sees two consecutive vertices of $A$. In the second case, each point of $\eta(e)$ which sees $t_{1}$ must see $a_{4}$ and $a_{1}$, while each point of $\eta(e)$ which sees $t_{2}$ must see $a_{4}$ and $a_{3}$. By the $\eta$-symmetry of $S$, this forces each point of $e$ to see either $a_{1}$ and $a_{4}$ or $a_{1}$ and $a_{2}$, again the desired result.

Now consider the situation in which $e \in A_{i}^{\prime}$ for some $i$, and by symmetry of our assumptions we may assume $i=1$. We select points $t_{1}$ and $t_{2}$ satisfying our hypothesis for the collection of edges $\left\{e, \xi(e), e_{L}, e_{R}, e_{U}, e_{D}\right\}$. As before, for an appropriate labeling, one of the following must occur: Either $t_{1} \in A_{4}$ and $t_{2} \in A_{2}$ or $t_{1} \in A_{1}$ and $t_{2} \in A_{3}$. 
There are three cases to investigate, depending on the classification of edge $e$.

Case A. Here we examine the case in which $e$ is a horizontal edge in $A_{1}^{\prime}$. Assume $e$ is a 'left' edge. Then $\xi(e)$ is a 'right' edge. If $t_{1} \in A_{4}$ and $t_{2} \in A_{2}$, then $\operatorname{conv}\left\{t_{2} \cup \xi(e)\right\} \subseteq S$. By symmetry, $\operatorname{conv}\left\{\xi\left(t_{2}\right) \cap e\right\} \subseteq S$, and in particular $e$ sees some point of $L \cap A_{1}$. The same holds if $t_{1} \in A_{1}$ and $t_{2} \in A_{3}$. But since $L_{+} \cap R_{+} \cap S$ is convex in horizontal direction and $\eta$-symmetric, it follows that $e$ sees both $a_{4}$ and $a_{1}$ via $S$, the desired result. Symmetrically, if $e$ is a 'right' edge, $e$ sees both $a_{2}$ and $a_{3}$ via $A$.

Case B. Now we assume that $e$ is a 'down' edge in $A_{1}^{\prime}$ and either preceded by a 'left' edge or followed by a 'right' edge (or both). If $e$ is preceded by a 'left' edge $f$, then by Case A each point of $f$ and in particular the top point of $e$ sees via $S$ both $a_{4}$ and $a_{1}$. From observation (3), it follows that every point of $e$ sees both $a_{4}$ and $a_{1}$ via $S$. Similarly, if $e$ is followed by a 'right' edge, the bottom point of $e$ sees both $a_{2}$ and $a_{3}$. Hence every point of $e$ sees both $a_{2}$ and $a_{3}$ via $S$.

Case C. It remains to consider the case in which $e$ is a 'down' edge in $A_{1}^{\prime}$ which is preceded by a 'right' edge and followed by a 'left' edge. For convenience of notation, let $e=[a, b]$ where $a$ is above $b$. Because $e_{L}$ is the first edge in $A_{1}^{\prime}, a \notin L$. Similarly, $b \notin D$. Recall that every point of the 'down' edges $e$ and $\xi(e)$ (which may coincide) sees via $S$ either $t_{1}$ or $t_{2}$. By the $\xi$-symmetry of $S$, every point of $e$ and $\xi(e)$ sees via $S$ either $\xi\left(t_{1}\right) \equiv s_{1}$ or $\xi\left(t_{2}\right) \equiv s_{2}$. There exist points $x_{1}$ and $x_{2}$ on $e$ such that $x_{1}$ sees via $S$ both $t_{1}$ and $t_{2}$ while $x_{2}$ sees via $S$ both $s_{1}$ and $s_{2}$. Without loss of generality, we may assume that $t_{1} \in A_{4}$ and $t_{2} \in A_{2}$. Hence $s_{1} \in A_{3}$ and $s_{2} \in A_{1}$. Observe that none of the segments $\left[a, t_{1}\right],\left[a, s_{2}\right],\left[b, s_{1}\right],\left[b, t_{2}\right]$ lie in $S$. (See Figure 4.) Both $a$ and $x_{1}$ see $t_{2}$ via $S$, so $\operatorname{conv}\left\{a, x_{1}, t_{2}\right\} \subseteq S$. In particular, every point of $\left[a, x_{1}\right]$ sees some point of $R \cap A_{2}$, and by observations (2) and (3), it follows that every point of $\left[a, x_{1}\right]$ sees both $a_{2}$ and $a_{3}$ via $S$. Similarly, since both $b$ and $x_{2}$ see $s_{2}$ via $S$, we conclude that every point of $\left[x_{2}, b\right]$ sees both $a_{4}$ and $a_{1}$. If $x_{2}$ is above $x_{1}$ or if $x_{1}=x_{2}$, the argument is finished. If $x_{1}$ is above $x_{2}$, it remains to show that every point of $\left[x_{1}, x_{2}\right]$ sees two consecutive vertices of $A$, and it is helpful to consider two complementary subcases.

Subcase C1. Here we assume that $\left[t_{1}, t_{2}\right]$ meets the segment $\left[a_{4}, a_{1}\right]$. Because of $\boldsymbol{\theta} \in\left[t_{1}, t_{2}\right]$ and $\xi$-symmetry, $\left[t_{1}, t_{2}\right]$ as well as $\left[s_{1}, s_{2}\right]$ meet both 


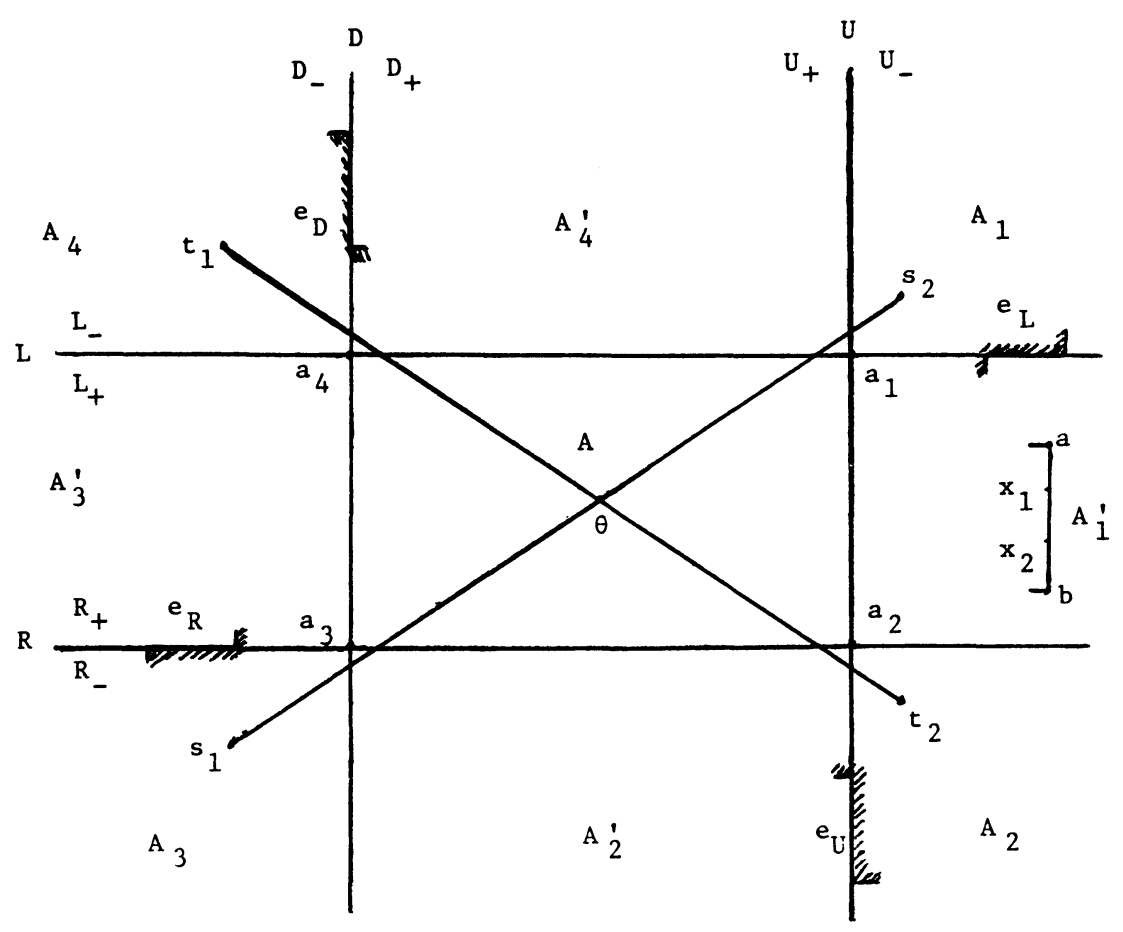

FIGURE 4

segments $\left[a_{4}, a_{1}\right]$ and $\left[a_{2}, a_{3}\right]$. Then $\left[x_{1}, t_{1}\right]$ meets $D$ in a point $y_{1} \in A_{4} \cap$ $D$ and $\left[x_{2}, s_{1}\right]$ meets $D$ in a point $y_{2} \in A_{3} \cap D$. By construction, $\left[x_{1}, t_{1}\right] \subseteq$ $S$ and $\left[x_{2}, s_{1}\right] \subseteq S$, so using observation (3), we conclude that $\left[x_{1}, y_{1}\right] \cup$ $\left[y_{1}, y_{2}\right] \cup\left[y_{2}, x_{2}\right] \cup\left[x_{2}, x_{1}\right] \subseteq S$. But $S$ is simply connected, so $\operatorname{conv}\left\{x_{1}, y_{1}, y_{2}, x_{2}\right\} \subseteq S$. Since $a_{3}$ and $a_{4}$ are on $\left[y_{1}, y_{2}\right]$, it follows that every point of $\left[x_{1}, x_{2}\right]$ sees via $S$ both $a_{3}$ and $a_{4}$.

Subcase C2. Here we assume that $\left[t_{1}, t_{2}\right]$ meets the segment $\left[a_{3}, a_{4}\right]$ in its relative interior. (See Figure 5.) Then $\left[\theta, t_{1}\right] \cap L \equiv p_{1} \in A_{4} \cap L,\left[\theta, s_{1}\right]$ $\cap R \equiv q_{2} \in A_{3} \cap R,\left[\theta, s_{2}\right] \cap L \equiv p_{2} \in A_{1} \cap L$, and $\left[\theta, t_{2}\right] \cap R \equiv q_{1} \in$ $A_{2} \cap R$. By construction, $\left[x_{2}, s_{2}\right]$ and $\left[x_{1}, t_{2}\right]$ lie in $S$. Thus by observations (2) and (3), $\left[\theta, p_{2}\right] \cup\left[\theta, q_{1}\right] \subseteq S$. By $\eta$-symmetry, $\left[\theta, p_{1}\right] \cup\left[\theta, q_{2}\right] \subseteq$ $S$ as well. Since $x_{1}$ sees both $t_{1}$ and $t_{2}$ via $s$, it follows from observation (3) that $x_{1}$ sees $p_{1}$ and $q_{1}$ via $S$ and also sees $a_{2}$ and $a_{3}$ via $S$. Similarly, we conclude that $x_{2}$ sees $p_{2}$ and $q_{2}$ via $S$ and also sees $a_{1}$ and $a_{4}$ via $S$. So we have again the situation of Figure 3 . Determine $\lambda \in[0,1]$ such that $a_{1}=\lambda p_{1}+(1-\lambda) p_{2}$. Then $a_{3}=\lambda q_{1}+(1-\lambda) q_{2}$. The conclusion drawn from Figure 3 in Case 1, Step 9, yields here that the point $x_{0} \equiv \lambda x_{1}+(1-\lambda) x_{2}$ sees both $a_{1}$ and $a_{3}$. Likewise, determine $\lambda^{\prime} \in[0,1]$ 


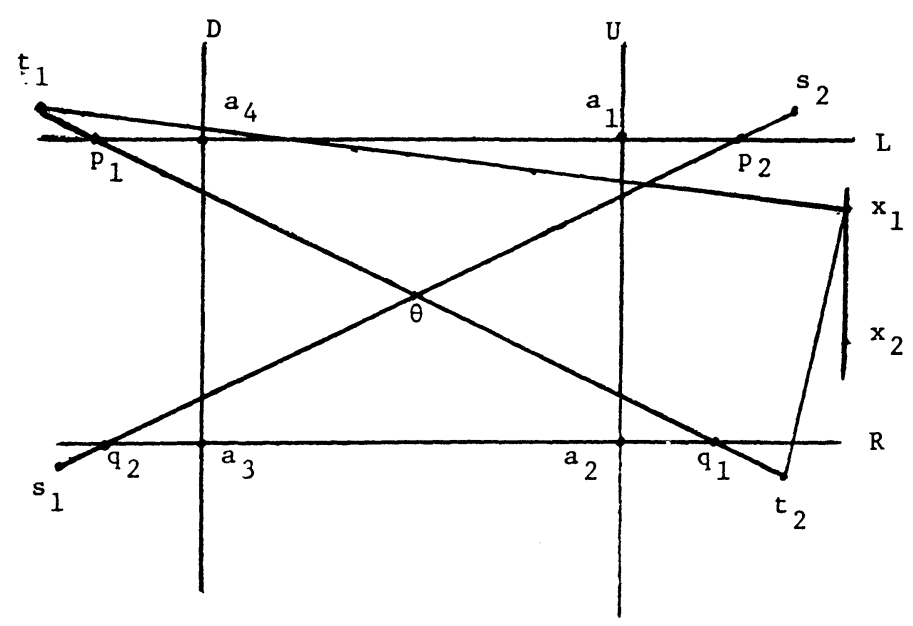

FIGURE 5

such that $a_{4}=\lambda^{\prime} p_{1}+\left(1-\lambda^{\prime}\right) p_{2}$. Then $a_{2}=\lambda^{\prime} q_{1}+\left(1-\lambda^{\prime}\right) q_{2}$, and as before the point $x_{0}^{\prime} \equiv \lambda^{\prime} x_{1}+\left(1-\lambda^{\prime}\right) x_{2}$ sees both $a_{2}$ and $a_{4}$. We have found that both $x_{1}$ and $x_{0}$ see $a_{3}$, and both $x_{0}$ and $x_{2}$ see $a_{1}$. Hence every point of $\left[x_{1}, x_{0}\right]$ sees $a_{3}$ and every point of $\left[x_{0}, x_{2}\right]$ sees $a_{1}$. Both $x_{1}$ and $x_{0}^{\prime}$ see $a_{2}$ and both $x_{0}^{\prime}$ and $x_{2}$ see $a_{4}$, so every point of $\left[x_{1}, x_{0}^{\prime}\right]$ sees $a_{2}$ and every point of $\left[x_{0}^{\prime}, x_{2}\right]$ sees $a_{4}$. In conclusion, every point of $\left[x_{1}, x_{2}\right]$ sees via $S$ either $a_{2}$ or $a_{4}$, and every point of $\left[x_{1}, x_{2}\right]$ sees via $S$ either $a_{1}$ or $a_{3}$. We have the required result in Subcase C2.

We have proved that each boundary point of $S$ sees via $S$ two consecutive vertices from $\left(a_{1}, a_{2}, a_{3}, a_{4}, a_{1}\right)$. Therefore, each boundary point of $S$ sees via $S$ either $a_{1}$ or $a_{3}$. By Lemma 1, each point of $S$ sees via $S$ either $a_{1}$ or $a_{3}$, and $S$ is a union of two starshaped sets in Case 2. This finishes the proof of Theorem 1 .

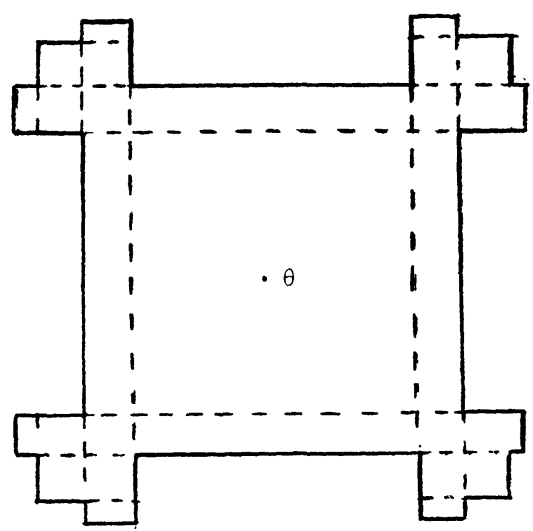

FIGURE 6 
To see that the number 6 in Theorem 1 is best possible, consider the following example.

EXAMPLE 3. Let $S$ be the simply connected set in Figure 6. Set $S$ is symmetric with respect to the $x$ and $y$ axes, and for every set $E$ consisting of 5 or fewer edges of $S$, there correspond points $t_{1}$ and $t_{2}$ collinear with $\theta$ such that every point in $\bigcup\{e: e$ in $E\}$ is visible via $S$ from $t_{1}$ or $t_{2}$. (Of course, $t_{1}$ and $t_{2}$ are not necessarily symmetric with respect to the origin.) However, $S$ is not a union of two starshaped sets.

We conclude the paper with an example adapted from [5, pp. 11-12] which reveals that no finite Krasnosel'skii number exists to characterize arbitrary unions of two or more compact starshaped sets, even in the plane. Hence the Krasnosel'skii-type result (2) in our introduction ([2, Theorem 2]) is best possible. Moreover, some restrictions like those in Theorem 1 must be imposed on our sets to obtain better results.

ExAmple 4. Let $n$ be fixed, $n \geq 2$. For $1 \leq i \leq 2 n$, let $C_{i}, C_{i}^{\prime}$ be arcs on the unit circle defined in polar coordinates as follows:

$$
\begin{aligned}
& C_{i}=\left\{(1, \theta):(i-n+1) \frac{\pi}{2 n} \leq \theta \leq(i+n-1) \frac{\pi}{2 n}\right\}, \\
& C_{i}^{\prime}=\left\{(1, \theta):(i+n+1) \frac{\pi}{2 n} \leq \theta \leq(i+3 n-1) \frac{\pi}{2 n}\right\} .
\end{aligned}
$$

Let $a_{i}$ and $b_{i}$ denote the endpoints of $C_{i}$, with $a_{i}^{\prime}$ and $b_{i}^{\prime}$ the endpoints of $C_{i}^{\prime}$. Finally, let $S_{n}=\bigcup\left\{\operatorname{conv} C_{i} \cup \operatorname{conv} C_{i}^{\prime}: 1 \leq i \leq 2 n\right\}$. (Figure 7 illustrates set $S_{n}$ when $n=2$.)

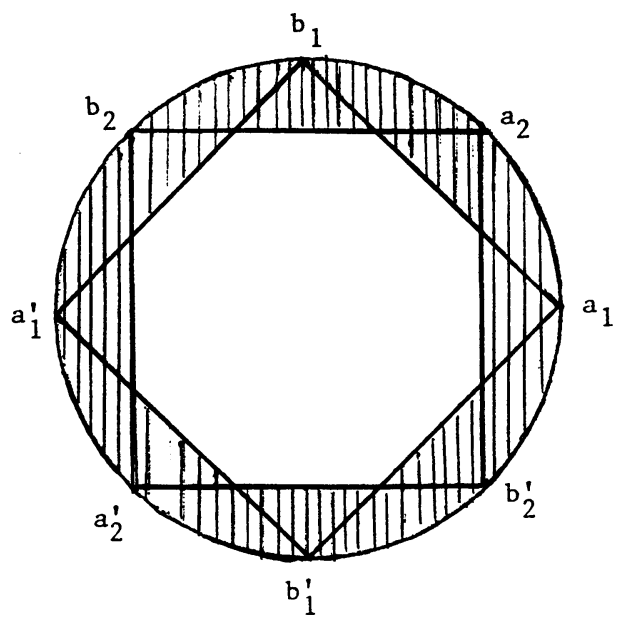

FIGURE 7 
Using an argument from [5, p. 11], every $2 n-1$ of the pairs of arcs $C_{i}, C_{i}^{\prime}$ have a common pair of antipodal points. Hence for every $(2 n-1)$ member subset $T$ of $S_{n}$, there correspond two points $s, t$ (depending on $T$ ) such that each point of $T$ is visible via $S$ from $s$ or $t$. However, there is no line meeting all $4 n$ arcs, and it is not hard to show that there are no two points $s^{\prime}, t^{\prime}$ satisfying the condition above for the set of midpoints

$$
T^{\prime}=\left\{\frac{1}{2}\left(a_{i}+b_{i}\right), \frac{1}{2}\left(a_{i}^{\prime}+b_{i}^{\prime}\right): 1 \leq i \leq 2 n\right\} .
$$

Since $n$ is arbitrary, it is clear that no finite Krasnosel'skii number exists for the general case. Thus the result in [2, Theorem 2] is best possible.

The author would like to thank the referee for providing the improved and shortened version of the proof of Theorem 1, Case 1, and for carefully reworking the text and figures of Theorem 1, Case 2 so that an assertion in Subcase C2 could be justified efficiently.

\section{REFERENCES}

[1] Marilyn Breen, An example concerning unions of two starshaped sets in the plane, Israel J. Math., 17 (1974), 347-349.

[2] _ Clear visibility and unions of two starshaped sets in the plane, Pacific J. Math., (to appear).

[3] Ludwig Danzer and Branko Grünbaum, Intersection properties of boxes in $R^{d}$, Combinatorica, 2 (3) (1982), 237-246.

[4] Ludwig Danzer, Branko Grünbaum and Victor Klee, Helly's theorem and its relatives, Proc. Symposia in Pure Math., Vol. VII (Convexity) (1963), 101-180.

[5] Hugo Hadwiger, Hans Debrunner, and Victor Klee, Combinatorial Geometry in the Plane, Holt, Rinehart and Winston, New York, 1964.

[6] M. A. Krasnosel'skii, Sur un critère pour qu' un domain soit étoilé, Math. Sb., 19 (61) (1946), 309-310.

[7] J. F. Lawrence, W. R. Hare, Jr., and John W. Kenelly, Finite unions of convex sets, Proc. Amer. Math. Soc., 34 (1972), 225-228.

[8] Steven R. Lay, Convex Sets and Their Applications, John Wiley, New York, 1982.

[9] Godfried T. Toussaint and Hossan El-Gindy, Traditional galleries are star-shaped if every two paintings are visible from some common point, Amer. Math. Monthly, (to appear).

[10] F. A. Valentine, Convex Sets, McGraw Hill, New York, 1964.

Received February 21, 1984.

UNIVERSITY OF OKLAHOMA

NORMAN, OK 73019 



\section{PACIFIC JOURNAL OF MATHEMATICS EDITORS}

V. S. VARAdarajan (Managing Editor)
University of California
Los Angeles, CA 90024
CHARLES R. DePrima
California Institute of Technology
Pasadena, CA 91125
R. FINN
Stanford University
Stanford, CA 94305

V. S. VARADARAJAN (Managing Editor)

Hermann FlaschKa

University of Arizona

Tucson, AZ 85721

RAMESH A. GANGOlli

University of Washington

Seattle, WA 98195

ROBION KIRBY

University of California

Berkeley, CA 94720
C. C. MOORE

University of California

Berkeley, CA 94720

H. SAMELSON

Stanford University

Stanford, CA 94305

Harold Stark

University of California, San Diego

La Jolla, CA 92093

\section{ASSOCIATE EDITORS}
R. ARENS
E. F. BECKENBACH
B. H. NeumanN
F. WOLF
K. Yoshida (1906-1982)

\section{SUPPORTING INSTITUTIONS}

\author{
UNIVERSITY OF ARIZONA \\ UNIVERSITY OF BRITISH COLUMBIA \\ CALIFORNIA INSTITUTE OF TECHNOLOGY \\ UNIVERSITY OF CALIFORNIA \\ MONTANA STATE UNIVERSITY \\ UNIVERSITY OF NEVADA, RENO \\ NEW MEXICO STATE UNIVERSITY \\ OREGON STATE UNIVERSITY
}

UNIVERSITY OF OREGON UNIVERSITY OF SOUTHERN CALIFORNIA

STANFORD UNIVERSITY

UNIVERSITY OF HAWAII

UNIVERSITY OF TOKYO

UNIVERSITY OF UTAH

WASHINGTON STATE UNIVERSITY

UNIVERSITY OF WASHINGTON 


\section{Pacific Journal of Mathematics}

\section{Vol. 120, No. $1 \quad$ September, 1985}

Ulrich F. Albrecht, A note on locally $A$-projective groups $\ldots \ldots \ldots \ldots \ldots 1$

Marilyn Breen, A Krasnosel'skiü-type theorem for unions of two starshaped

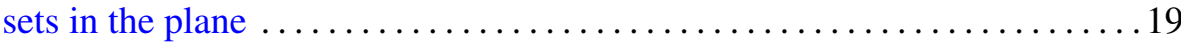

Anthony Carbery, Sun-Yung Alice Chang and John Brady Garnett,

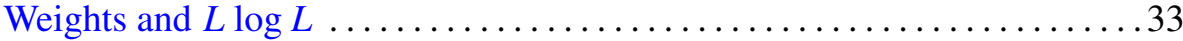

Joanne Marie Dombrowski, Tridiagonal matrix representations of cyclic

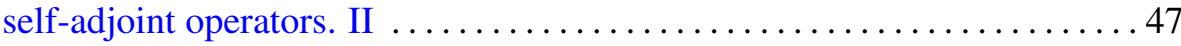

Heinz W. Engl and Werner Römisch, Approximate solutions of nonlinear random operator equations: convergence in distribution $\ldots \ldots \ldots \ldots \ldots 55$

P. Ghez, R. Lima and J. E. Roberts, $W^{*}$-categories $\ldots \ldots \ldots \ldots \ldots \ldots$

Barry E. Johnson, Continuity of homomorphisms of Banach $G$-modules . . 111 Elyahu Katz and Sidney Allen Morris, Free products of topological groups

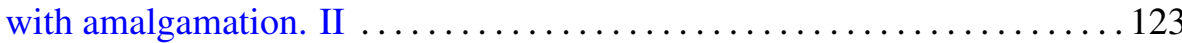

Neal I. Koblitz, $p$-adic integral transforms on compact subgroups of $\mathbf{C}_{p} \ldots 131$ Albert Edward Livingston, A coefficient inequality for functions of positive real part with an application to multivalent functions $\ldots \ldots \ldots 139$

Scott Carroll Metcalf, Finding a boundary for a Hilbert cube manifold

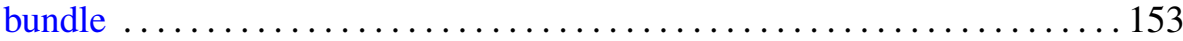

Jack Ray Porter and R. Grant Woods, When all semiregular $H$-closed extensions are compact ........................

Francisco José Ruiz and José Luis Torrea, A unified approach to Carleson measures and $A_{p}$ weights. II $\ldots \ldots \ldots \ldots \ldots \ldots \ldots \ldots \ldots \ldots \ldots \ldots \ldots$

Timothy DuWayne Sauer, The number of equations defining points in general position

John Brendan Sullivan, Universal observability and codimension one subgroups of Borel subgroups

Akihito Uchiyama, Extension of the Hardy-Littlewood-Fefferman-Stein inequality 